\title{
Retraction Note: Application of support vector neural network with variational mode decomposition for exchange rate forecasting
}

\author{
Yungao $\mathrm{Wu}^{1,2} \cdot$ Jianwei $\mathrm{Gao}^{1}$
}

Published online: 12 August 2021

(C) Springer-Verlag GmbH Germany, part of Springer Nature 2021

\section{Retraction to: Soft Computing (2018) 23:6995-7004 https://doi.org/10.1007/s00500-018-3336-1}

The authors have retracted this article due to a substantial flaw in the model and a serious error in the experimental execution, which renders the conclusions of this article incorrect and unreliable. In the proposed model, variational mode decomposition (VMD) is employed to decompose the exchange rate time series, while the whole time series (including the training set and the test set) is decomposed, which means the future information has been used in the decomposition process. In the experimental execution, the training set is used for model training, and the test set is used for model evaluation, while the training set and the test set are both adopted to train the proposed model, which means all the experimental results are incorrect and unreliable. The corresponding author, Yungao Wu, accepts full responsibility for the errors. The author Jianwei Gao stated that he was not aware of this publication.

Publisher's Note Springer Nature remains neutral with regard to jurisdictional claims in published maps and institutional affiliations.

The original article can be found online at https:// doi.org/10.1007/s00500-018-3336-1.

Yungao $\mathrm{Wu}$

wuyungao_2007@126.com

1 School of Economics and Management, North China Electric Power University, Beijing 102206, China

2 Department of Mathematical Sciences, Ordos Institution of Applied Technology, Ordos 017000, China 